\title{
Enhanced Bitwidth-Aware Register Allocation
}

\author{
Rajkishore Barik and Vivek Sarkar \\ IBM T.J. Watson Research Center
}

\begin{abstract}
Embedded processors depend on register files for performance, just like general-purpose processors in desktop and server systems. However, unlike general-purpose processors, the power consumption of register files poses a significant challenge for embedded processors, making it desirable for embedded processors to use as few registers as possible. Past research has indicated the potential for leveraging bitwidth analysis and bitwidth-aware register allocation to reduce register usage in embedded applications.

This paper makes the following contributions in evaluating and enhancing bitwidth-aware register allocation for embedded applications. First, we compare the Tallam-Gupta bitwidth analysis with an idealized limit study, and show significant opportunities for enhancements. Second, we show how bitwidth-aware register allocation can be enhanced by enhanced bitwidth analysis for scalar and array variables, and also by enhanced coalescing of variables. Third, we use our prototype implementation of bitwidth-aware register allocation in $g c c$ to compare the number of dynamic spill load/store instructions resulting from a) bitwidth-unaware allocation, b) bitwidth-aware allocation, c) enhanced bitwidth-aware allocation, and d) ideal profile-driven bitwidth-aware allocation. Our results show that our enhancements can reduce the number of dynamic spill load/store instructions to between $3 \%$ and $27 \%$ of the number obtained from the Tallam-Gupta algorithm.
\end{abstract}

\section{Introduction}

Embedded applications operate extensively on subword data values i.e., data values with narrower width than the standard data width (word size) supported by the underlying embedded processor e.g., 32 bits or 64 bits. As indicated by Tallam and Gupta 20, the register usage of embedded applications can be reduced by employing bitwidth-aware register allocation. A reduction in register usage can then be leveraged to reduce the power requirements of the embedded application on an embedded system [11. Similarly, bitwidth analysis has been used to reduce the number of registers needed in the context of silicon compilation [19] and reconfigurable architectures. The opportunities for reducing register usage are even more promising when moving to embedded processors with a 64-bit word size (compared to a 32-bit word size).

One cornerstone of bitwidth-aware register allocation lies in its underlying bitwidth analysis. Stephenson et al. introduced a bitwidth analysis for silicon compilation [19] and showed how it can be used to reduce the total number of

A. Mycroft and A. Zeller (Eds.): CC 2006, LNCS 3923, pp. 263-276 2006

(C) Springer-Verlag Berlin Heidelberg 2006 
register bits in the generated RTL. However, the silicon compilation problem differs from register allocation, because there is no notion of register spills in silicon compilation. The Tallam-Gupta algorithm addresses this problem more directly by providing a register allocation algorithm that is capable of packing multiple subwords into a single register. There is an underlying trade-off when performing bitwidth-aware register allocation viz., reducing the number of registers used can save power, but may introduce additional overhead for subword access. This trade-off is well suited to new embedded processors in which the overhead of subword access is reduced by direct hardware support, and where the benefits of using fewer registers is clearly visible in reduced power.

Another cornerstone lies in the coalescing heuristics employed by bitwidthaware register allocation. Unlike coalescing in conventional register allocation, it is permissible to coalesce two interfering variables in bitwidth-aware register allocation (provided that the sum of their bitwidths does not exceed the register word size). As shown in Figure 5, the estimates for coalescing provided in [20] can be improved significantly to perform a better coalescing of live ranges.

This paper makes the following contributions in evaluating and enhancing bitwidth-aware register allocation for embedded applications:

1. A limit study (Section 2) that compares compare the Tallam-Gupta bitwidth analysis algorithm [20] with dynamic profile-driven bitwidth information, and show significant opportunities for enhancements.

2. An enhanced bitwidth analysis algorithm (Section 3) that performs more detailed scalar analysis and array analysis for improved bitwidth information than in 20].

3. An enhanced coalescing algorithm (Section 4) that performs less conservative (more aggressive) coalescing than in [20].

4. Experimental results from a prototype implementation of bitwidth-aware register allocation in $g c c$ to compare the effect of the two main enhancements listed above.

Our results show that our enhancements can reduce the dynamic number of load/store instructions significantly, compared to the Tallam-Gupta bitwidthaware allocation algorithm. In the best case, our enhancements resulted in a reduction of the load/store instructions to $3 \%$ of the bitwidth-unaware case (for 6 registers). As can be seen in Table 4 the reductions delivered by our enhancements can also be smaller - the "worst" case is a reduction to $27 \%$ of the orginal dynamic number of load/store instructions. To the best of our knowledge this is the first study that reports on the dynamic number of load/store instructions (spill instructions) resulting from bitwidth-aware register allocation. (The results reported in 20] were for static register requirements and static numbers of live ranges and interference graph nodes.)

Davidson and Jinturkar [8] proposed a compiler optimization that exploits narrow width data. They used memory coalescing to improve cache performance of a program. It will be interesting to see how the approach advocated in this paper for register allocation could be extended in the future to improve cache performance. 
The rest of this paper is organized as follows. Section 2 introduces metrics such as the Active Compression Factor (ACF) to compare the Tallam-Gupta bitwidth analysis with an idealized limit study, and thereby show significant opportunities for enhancements. Section 3 shows how bitwidth-aware register allocation can be improved by enhanced analysis of scalar and array variables. Section 4 shows how additional improvements can be obtained by enhancements to the coalescing algorithm and its underlying framework. Section 5 reports on our experimental results obtained from our prototype implementation of bitwidth-aware register allocation. Finally, Section 6 contains our conclusions.

\section{Limit Study of Bitwidth Usage}

Our first step in studying bitwidth-aware register allocation was to perform a limit study that compares the bitwidth usage computed by the static compiletime bitwidth analysis algorithm in [20] with dynamic bitwidth information obtained from an execution profile. The infrastructure used for this study was based on the GCC compiler, as depicted in Figure 1. The register allocation phase in gcc was modified to accept input from the box labeled "Bitwidth analysis", which can either generate compile-time or profile-driven bitwidth information. In general, the width of a variable at a program point can be represented by three parts: a leading part of unused bits, a middle part of active bits, and a trailing part of unused bits. We implemented the Tallam-Gupta algorithm in the GCC compiler to obtain this information for the compile-time case. For the profile-driven case, we instrumented the code generated by GCC so as to perform a "logical or" of the values dynamically assigned to a each variable. The major motivation for performing the limit study is that the prior work by Gupta and Tallam reported static benefits of bitwidth-aware register allocation (fewer registers used, smaller cliques in the interference graph), but did not provide any indication of what additional opportunities remain for improved bitwidth analysis.

The benchmarks used in this paper were all taken from the Bitwise benchmark set 1], so as to be representative of embedded applications. Our evaluation was performed on 9 out of the 15 programs in the full benchmark set. The following five programs were not used because they did not contain a return value, thereby making it possible for gcc to optimize away the entire program as dead code bilint, levdurb, motiontest, sha, softfloat. In addition, the life program was not used, because the Bitwise benchmark set already contains a newlife program which is very similar to life. All experiments were performed using the -O3 option and the - param max-unroll-times $=0$ option $^{1}$ with version 4.1 of gcc targeted to the x86 platform.

Table 1 lists the total number of variables (pseudoregisters) available for register allocation in each benchmark, followed by the number of variables that were

${ }^{1}$ This option disables loop unrolling. Loop unrolling can create more candidates for register allocation, but the relative impact of unrolling depends on the benchmark so it was disabled. 


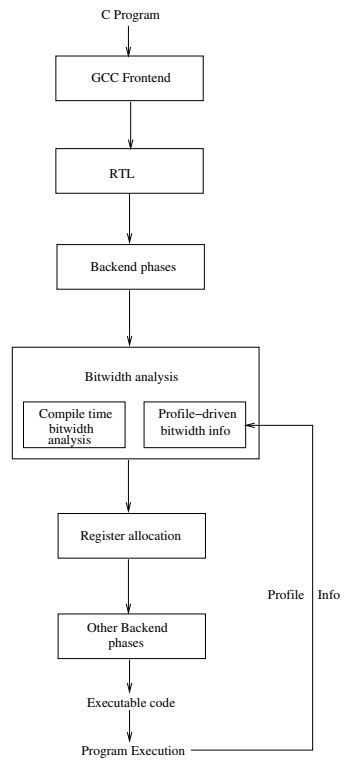

Fig. 1. GCC modification for limit study

identified to have varying bitwidth by static analysis, and next by the number of variables that were identified to have variable bitwidth by profile information. The results in the table indicate that there is opportunity for significant improvement in compile-time bitwidth analysis, compared to the static analysis obtained from the Tallam-Gupta algorithm.

We now introduce another metric called the active compression factor (ACF) to measure the effectiveness of the bit sensitive analysis. Let $A B_{i j}$ denote the number of active bits in register operand $j$ at statement $i$ (obtained either from static analysis or from profile information), and $T B_{j}$ denote the number of total bits in register operand $j$ (in other words, the statically defined size of $j$ ). Let

Table 1. Comparison of compile-time and profile-driven bitwidth analysis: Number of and percentage of variables with bitwidth $<32$ bits

\begin{tabular}{|c||r|r|r|}
\hline & Total \# variables & \multicolumn{2}{|c|}{ Total \# and \% of variables with variable bitwidth } \\
\hline Benchmark & & (Compiler analysis) & (Profile-driven) \\
\hline \hline adpcm & 26 & $20(76.92 \%)$ & $25(96.15 \%)$ \\
bubblesort & 20 & $11(55.00 \%)$ & $20(100.00 \%)$ \\
convolve & 8 & $6(75.00 \%)$ & $7(87.50 \%)$ \\
edge_detect & 107 & $20(18.69 \%)$ & $76(71.02 \%)$ \\
histogram & 29 & $16(55.17 \%)$ & $23(79.31 \%)$ \\
jacobi & 36 & $13(36.11 \%)$ & $23(63.88 \%)$ \\
median & 33 & $9(27.27 \%)$ & $26(78.78 \%)$ \\
mpegcorr & 30 & $13(43.33 \%)$ & $21(70.00 \%)$ \\
newlife & 62 & $19(30.64 \%)$ & $48(77.41 \%)$ \\
\hline
\end{tabular}


$F R E Q_{i}$ denote the dynamic frequency of statement $i$. We define the active compression factor as follows:

$$
A C F=\frac{\sum_{i \in I N S N} \sum_{j \in R E G O P E R A N D} F R E Q_{i} * T B_{j}}{\sum_{i \in I N S N} \sum_{j \in R E G O P E R A N D} F R E Q_{i} * A B_{i j}}
$$

Note that $A C F$ must be $\geq 1$ since $T B_{j} \geq A B_{i j}$.

Table 2 shows $A C F$ values for the compile-time and profile-driven cases. The same execution profile information is used for the $F R E Q_{i}$ values in both cases - the difference lies in the computation of the $A B_{i j}$ values. A larger $A C F$ value indicates a greater opportunity for bitwidth-aware register allocation. The results in Table 2 show ACF values in the range of 1.0 to 1.37 for the compiletime case, and in the range of 1.45 to 3.90 for the profile-driven case. Once again, this shows opportunity for improved bitwidth analysis, compared to the results obtained from the Tallam-Gupta algorithm.

Table 2. Active Compression Factor (ACF) comparison across static and profile-driven bitwidth analysis without loop unrolling

\begin{tabular}{|c||r|r|}
\hline Benchmark & $\begin{array}{r}\text { Compile-time } \\
\text { compression }\end{array}$ & $\begin{array}{r}\text { Profile-driven } \\
\text { compression }\end{array}$ \\
\hline \hline adpcm & 1.37 & 3.39 \\
bubblesort & 1.21 & 3.90 \\
convolve & 1.00 & 3.05 \\
edge_detect & 1.04 & 2.26 \\
histogram & 1.10 & 2.09 \\
jacobi & 1.00 & 1.67 \\
median & 1.01 & 2.14 \\
mpegcorr & 1.03 & 1.94 \\
newlife & 1.05 & 2.67 \\
\hline
\end{tabular}

\section{Enhanced Bitwidth Analysis}

In this section, we outline two key enhancements that we made to the bitwidth analysis in the Tallam-Gupta algorithm, both of which were motivated by the opportunities identified by the limit study in the previous section.

1. Enhanced Scalar Analysis. The Tallam-Gupta algorithm performs a data flow analysis that includes a forward zero bit section analysis and a backward dead bit section analysis. We added a recurrence analysis (using the algorithm in [3]) that can identify general induction variables and other patterns with closed form solutions. This is more general than the scalar range analysis presented by Stephenson et al [19].

2. Enhanced Array Analysis. A key limitation of the Tallam-Gupta algorithm is that it performs no analysis of array variables. We added an array range analysis that tracks the values being assigned to arrays, and integrates the array analysis with the enhanced scalar analysis. This enhancement performs 
a flow-insensitive analysis of all accesses to an array variable. In the future, we plan to implement a flow-sensitive version, based on the Array SSA form algorithm for element-level constant propagation [13].

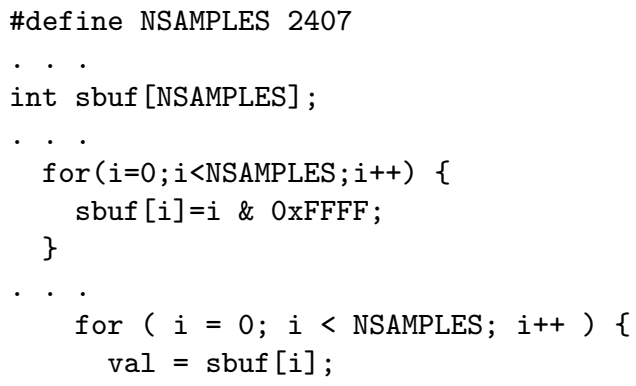

Fig. 2. Code fragment from BITWISE adpcm benchmark

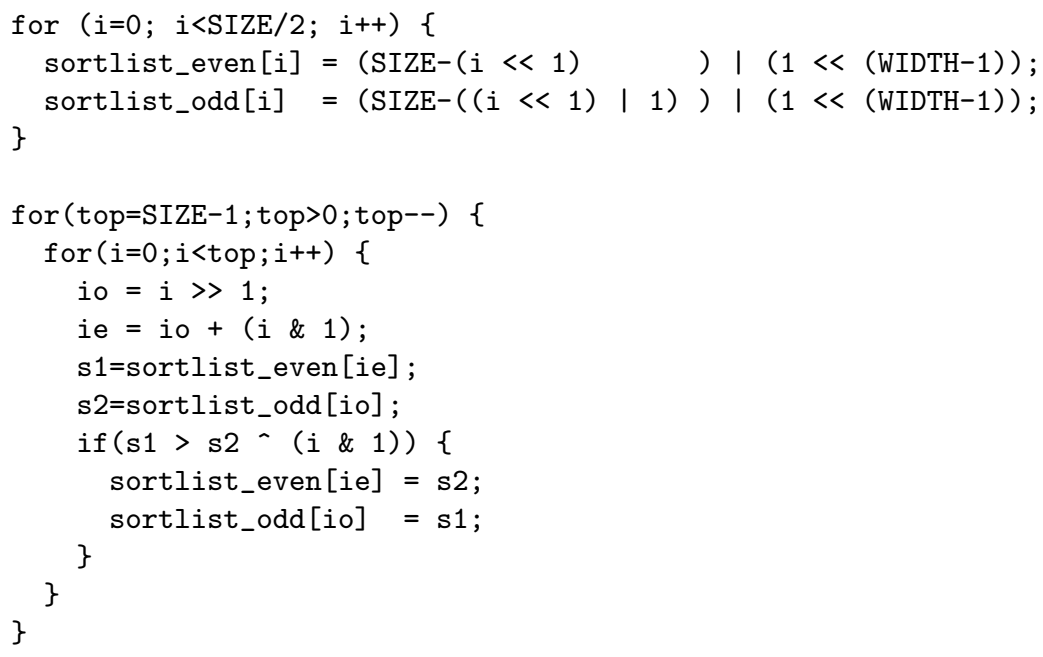

Fig. 3. Code fragment from BITWISE bubblesort benchmark

We use two code examples to illustrate the benefits of these two enhancements, and how they are used in conjunction with each other. Figure 2 contains a code fragment from the Bitwise adpcm benchmark. While it may not be standard practice in general, it is common practice in embedded applications for loop iterations to be bounded by constants defined in the program. When analyzing the expression, i \& OxFFFF, our enhanced analysis determines that variable $i$ must be in the range, $0 \ldots 2406$. Further, the constant 0xFFFF value has a bitwidth of 16 bits. Hence, each element assigned to the sbuf array has a lower bound of 0 , and an 
upper bound of $\min (2406,65535)=2406$, or a maximum bitwidth of 12 bits (The min function is applied when a bitwise-and operator is being analyzed.). Scalar variable val is then bounded by a maximum bitwidth of 12 bits.

Figure 3 contains a code fragment from the Bitwise bubblesort benchmark. There are two static definitions each for arrays sortlist_even and sortlist_odd. However, the values of s2 and s1 that appear in the right-handside of the second pair of definitions originate from the same arrays. Therefore, our data flow analysis determines that the bitwidth of the array elements must be bounded by their initial definition i.e., 17 bits.

\section{Enhanced Coalescing for Bitwidth-Aware Register Allocation}

In this section, we outline improvements in the coalescing heuristics used in bitwidth-aware register allocation. Figure 4 contains a summary of the TallamGupta bitwidth-aware register allocation algorithm, as presented in Figure 13 in 20. The key step that implements the coalescing heuristic is Step 7. As shown later in our experimental results, enhancements to the coalescing heuristic can have a significant impact on the effectiveness of bitwidth-aware register allocation. Note that the coalescing step in bitwidth-aware register allocation is different from coalescing in classical register allocation. In classical register allocation, two non-interfering variables can be coalesced so as to use the same register. Bitwidthaware register allocation allows two interfering variables to be coalesced provided the sum of their bitwidths does not exceed the register word size.

We now discuss three key characteristics of the Tallam-Gupta algorithm, and outline how they were extended/replaced in our algorithm:

1. Coalescing is performed conservatively in the Tallam-Gupta algorithm i.e., coalescing is restricted to cases when the node created by coalescing two nodes has fewer than $k$ neighbors with degree of $k$ or more (where $k$ is the number of registers available for allocation). However, our experimental results show that this restriction is too conservative in many cases, so we use aggressive coalescing algorithm originally proposed by Chaitin [7.

2. If nodes $A$ and $B$ are coalesced, and both have an edge to another node, $C$, it is necessary to compute a new label for the edge from the new coalesced node, $A B$ to $C$. As discussed in Section 4.1 below, the heuristic used in the Tallam-Gupta algorithm can result in edge labels that are unnecessarily large, thereby precluding some possible coalescing heuristics. Our algorithm performs a more precise update of the edge label.

3. The priority function for live ranges used in the Tallam-Gupta algorithm for selecting nodes in Step 3 is defined as follows:

$$
\begin{aligned}
\text { Priority }(l r) & =\frac{\text { Estimated Load/Store Savings }}{\text { Live Range Area }} \\
& =\frac{\text { Estimated Load/Store Savings }}{\sum_{\text {Program point } p \text { width }(l r, p)}}
\end{aligned}
$$


However, our experience has shown that this priority function often favors short-lived live ranges which have a small area, even though they may not offer a large savings in load/store instructions. Our enhancement was to remove the denominator term in the priority function, so that all live ranges are prioritized (largest-first) according to the estimated absolute load/store savings.

These three enhancements together result in the "Enhanced Coalescing" experimental results presented in Section 5 .

1. Construct interference graph.

2. Label edges with interference widths.

3. Construct prioritized node list.

4. while node list $\neq \phi$ do

5. Get a node, say $n$, from prioritized node list.

6. for each node $a$ in $n$ 's adjacency list do

7. Attempt coalescing a with $n$. // Coalescing heuristic

8. If successful, update interference graph and prioritized node list.

$9 . \quad$ end for

10. end while

11. Replace each coalesced variable set with a new name.

12. Introduce intravariable moves.

13. Perform graph coloring register allocation

Fig. 4. Tallam-Gupta Bitwidth-aware Register Allocation

\subsection{Updating Edge Labels After Coalescing}

As mentioned earlier, the update of edge labels after coalescing can be unnecessarily large in the Tallam-Gupta algorithm. Each edge $(X, Y)$ is labeled with an ordered pair, $\left(X_{y}, Y_{x}\right)$, such that $X_{y}$ and $Y_{x}$ estimate the maximum widths of $X$ and $Y$ at a program point corresponding to the maximum interference width of $X$ and $Y$. A key constraint is that nodes $X$ and $Y$ cannot be coalesced if $X_{y}+Y_{x}$ is larger than the register word size.

Let us consider the interference graph shown in Figure [5] as an example in which we attempt to coalesce nodes $A$ and $B$. The upper right section of Figure 5 shows the actual number of bits required by variables $A, B$ and $C$ in the instruction stream. The Tallam-Gupta algorithm uses an Estimated Maximum Interference Width (EMIW) computation that results in the edge label $(28,8)$ as shown on the lower left of Figure 5 (Refer to $E_{\text {int }}$ in [20]). In this case, node $A B$ cannot be coalesced later with $C$, since $28+8=36$ is larger than the 32-bit word size assumed in this example. However, if we use our enhanced estimates as illustrated in the lower right of Figure 5. the resulting edge label is $(21,8)$, which would permit nodes $A B$ and $C$ to be coalesced next. The key point is that the Tallam-Gupta algorithm conservatively estimates the bitwidth of $A B$ after coalescing to be 28 , though it should actually be 21 . 

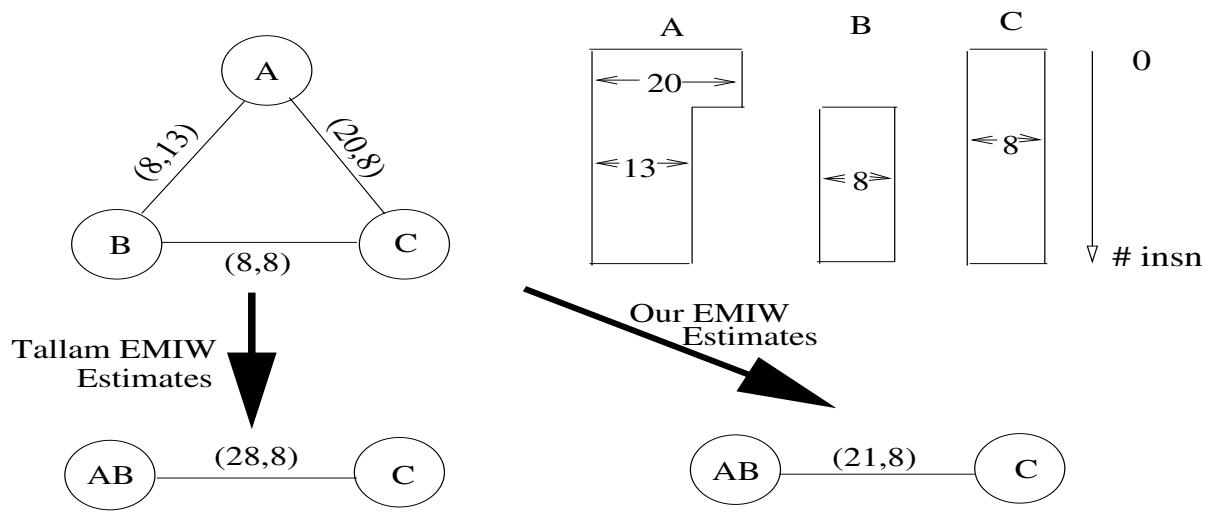

Fig. 5. Example Interference Graph

The details of our more precise estimate of edge labels can be obtained from the equations listed below, which we used to add to the equations for Case II in Figure 7 of [20]. NODEMAX denotes the maximal width of a node across all program points. We have also proved that this more precise estimation satisfies the intermediate value theorem in 20]. The proofs are included in Appendix $\mathrm{A}$,

$$
\begin{aligned}
& E_{1}=A_{b}+B_{a}+\operatorname{NODEMAX}(C) \\
& E_{2}=A_{c}+C_{a}+\operatorname{NODEMAX}(B) \\
& E_{3}=C_{b}+B_{c}+\operatorname{NODEMAX}(A) \\
& E_{4}=B_{a}+C_{a}+\operatorname{MAX}\left(A_{b}, A_{c}\right) \text { if } E_{\text {min }}=E_{a} \text { and } E_{4} \geq E_{\text {min }} \\
& E_{5}=A_{b}+C_{b}+\operatorname{MAX}\left(B_{a}, B_{c}\right) \text { if } E_{\text {min }}=E_{b} \text { and } E_{5} \geq E_{\text {min }} \\
& E_{6}=A_{c}+B_{c}+\operatorname{MAX}\left(C_{a}, C_{b}\right) \text { if } E_{\text {min }}=E_{c} \text { and } E_{6} \geq E_{\text {min }}
\end{aligned}
$$

$\operatorname{EMIW}(A, B, C)=\operatorname{MIN}\left(E_{\text {int }}, E_{1}, E_{2}, E_{3}, E_{4}, E_{5}, E_{6}\right)$

$$
\begin{aligned}
& \left(A B_{c}, C_{a b}\right)=\left(A_{b}+B_{a}, \operatorname{NODEMAX}(C)\right) \text { if } \operatorname{EMIW}(A, B, C)=E_{1} \\
& \left(A B_{c}, C_{a b}\right)=\left(A_{c}+\operatorname{NODEMAX}(B), C_{a}\right) \text { if } \operatorname{EMIW}(A, B, C)=E_{2} \\
& \left(A B_{c}, C_{a b}\right)=\left(B_{c}+\operatorname{NODEMAX}(A), C_{b}\right) \text { if } \operatorname{EMIW}(A, B, C)=E_{3} \\
& \left(A B_{c}, C_{a b}\right)=\left(B_{a}+\operatorname{MAX}\left(A_{b}, A_{c}\right), C_{a}\right) \text { if } \operatorname{EMIW}(A, B, C)=E_{4} \\
& \left(A B_{c}, C_{a b}\right)=\left(A_{b}+M A X\left(B_{a}, B_{c}\right), C_{b}\right) \text { if } \operatorname{EMIW}(A, B, C)=E_{5} \\
& \left(A B_{c}, C_{a b}\right)=\left(A_{c}+B_{c}, M A X\left(C_{a}, C_{b}\right)\right) \text { if } \operatorname{EMIW}(A, B, C)=E_{6}
\end{aligned}
$$

\section{$5 \quad$ Experimental Results}

In this section, we report on experimental results obtained from our prototype implementation of bitwidth-aware register allocation based on gcc. Figure 6] 


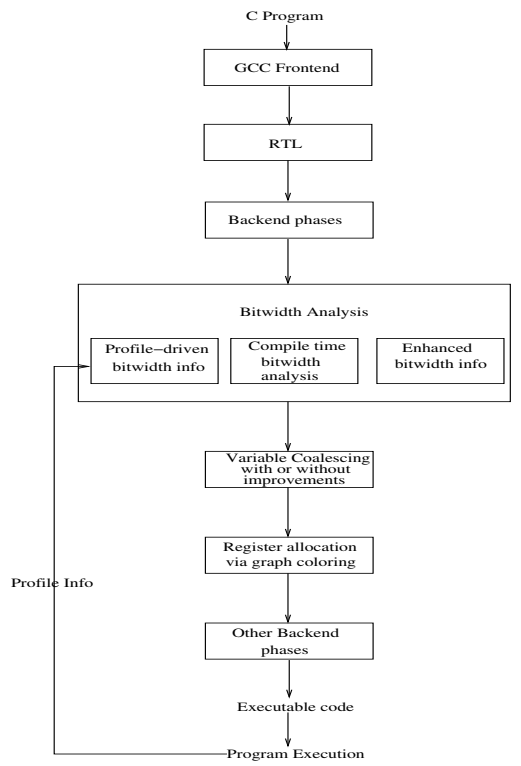

Fig. 6. GCC modification for register allocation

depicts how the bitwidth-aware register allocator is inserted into the phases of the gcc compiler. A standard graph coloring register allocator [7] was used instead of GCC's local and global register allocator. Note that we now have three options for Bitwidth Analysis — the Tallam-Gupta algorithm, enhanced bitwidth analysis, and profile-driven information. The enhanced analysis results were obtained by our implementation of the enhanced scalar and array analysis outlined in Section [3. Also, there are two options for Variable Coalescing the Tallam-Gupta algorithm or the enhanced coalescing algorithm outlined in Section 4 .

The experimental results reported in this section will be used to compare five different cases:

1. Bitwidth-Unaware - a standard graph coloring algorithm is used with no support for bitwidth-aware register allocation.

2. + Bitwidth-Aware - enhancement of the previous case by using the TallamGupta bitwidth-aware register allocation.

3. + Enhanced Coalescing — addition of the enhanced coalescing techniques introduced in Section 4 of this paper.

4. + Enhanced Bitwidth - addition of the enhanced scalar and array bitwidth analysis techniques introduced in Section 3 of this paper.

5. + Profiled Bitwidth — like the previous case, but with profiled bitwidth information from the limit study used instead of statically analyzed bitwidth information.

The benchmark programs being used in this section are the same as those that were used for the limit study described in Section 2 
Table 3. Comparison of number of coalesce node-pairs with different levels of bitsensitive register allocation for the number of available registers $=8$

\begin{tabular}{|c||r|r|r|r|}
\hline Benchmarks & $\begin{array}{r}\text { Bitwidth-Aware } \\
\text { (Tallam-Gupta) }\end{array}$ & $\begin{array}{r}\text { Enhanced } \\
\text { Coalescing }\end{array}$ & $\begin{array}{r}\text { Enhanced } \\
\text { Bitwidth }\end{array}$ & $\begin{array}{r}\text { Profiled } \\
\text { Bitwidth }\end{array}$ \\
\hline \hline adpcm & 0 & 7 & 15 & 18 \\
bubblesort & 1 & 1 & 12 & 12 \\
convolve & 0 & 0 & 2 & 2 \\
edge_detect & 0 & 0 & 25 & 64 \\
histogram & 1 & 1 & 15 & 15 \\
jacobi & 0 & 0 & 15 & 16 \\
median & 0 & 0 & 16 & 17 \\
mpegcorr & 0 & 0 & 10 & 13 \\
newlife & 0 & 2 & 40 & 41 \\
\hline
\end{tabular}

As can be seen in Figure 6, the same register allocator based on graph coloring is used in all cases. Therefore, the only way for the bitwidth-aware heuristics to demonstrate an improvement compared to bitwidth-unaware allocation, is for the heuristics to perform some coalescing of nodes.

Table 3 reports the number of node-pairs coalesced when processing all nine benchmark programs for number of available registers 8 . Note that the coalescing pre-pass for Tallam-Gupta depends on the number of available registers (conservative coalescing) whereas our modified approach does not (aggressive coalescing). The results show that our combined heuristic (Case 4 above) performs significantly more coalescing than the Tallam-Gupta algorithm.

Next, Table 4 compares the number of dynamic load/store instructions arising from register spills for the five different cases. Each row represents the case for a certain number of available registers, and each entry represents the sum of the dynamic load/store spill instructions for the nine benchmarks.

As seen in Table 4, the Tallam-Gupta algorithm had zero impact on reducing the number of dynamic load/store spill instructions, for the cases studied, and essentially yielded the same dynamic spill load/store instruction count as the bitwidth-unaware. However, the techniques introduced in our paper (cases 3

Table 4. Comparison of dynamic spill load/store instructions with different levels of bit-sensitive register allocation for the number of available registers $=8$

\begin{tabular}{|c||r|r|r|r|r|}
\hline $\begin{array}{c}\text { Number of } \\
\text { registers }\end{array}$ & $\begin{array}{r}\text { Bitwidth-Unaware } \\
\text { (Standard Coloring) }\end{array}$ & $\begin{array}{r}\text { + Bitwidth-Aware } \\
\text { (Tallam-Gupta) }\end{array}$ & $\begin{array}{r}\text { + Enhanced } \\
\text { Coalescing }\end{array}$ & $\begin{array}{r}\text { + Enhanced } \\
\text { Bitwidth }\end{array}$ & $\begin{array}{r}\text { + Profiled } \\
\text { Bitwidth }\end{array}$ \\
\hline \hline 4 & 2427150 & 2427150 & $1973769(81.00)$ & $669469(27.00)$ & $622421(25.00)$ \\
6 & 836687 & 836687 & $267324(31.00)$ & $26443(3.00)$ & $18953(2.00)$ \\
8 & 58633 & 58633 & $36967(63.00)$ & $6909(11.00)$ & $5370(9.00)$ \\
10 & 19581 & 19581 & $19571(99.00)$ & $3342(17.00)$ & $1803(9.00)$ \\
12 & 9945 & 9945 & $9945(100.00)$ & $1824(18.00)$ & $527(5.00)$ \\
14 & 6378 & 6378 & $6378(100.00)$ & $548(8.00)$ & $0(0)$ \\
16 & 4860 & 4860 & $4860(100.00)$ & $10(0)$ & $0(0)$ \\
18 & 3342 & 3342 & $3342(100.00)$ & $0(0)$ & $0(0)$ \\
\hline
\end{tabular}


and 4 above) reduced the dynamic spill load/store instruction count to $3 \%$ to $27 \%$ of the bitwidth-unaware case. This is a significant reduction.

\section{Conclusions and Future Work}

In this paper, we studied the problem of enhancing bitwidth-aware register allocation. Our limit study showed significant opportunities for improvement, compared to the algorithm pioneered by Tallam and Gupta. We used our prototype implementation of bitwidth-aware register allocation in gcc to compare the dynamic number of load/store instructions) resulting from a) bitwidth-unaware allocation, b) bitwidth-aware allocation, c) enhanced bitwidth-aware allocation with improved bitwidth analysis and improved coalescing, and d) ideal profiledriven bitwidth-aware allocation. Our results show that our enhancements can reduce the dynamic number of spill load/store instructions to $3 \%$ to $27 \%$ of the number obtained from the Tallam-Gupta algorithm.

In future, we would like to study the overhead of bit-aware register allocation (number of extra instructions added), effect on run-time performance and energy reduction.

\section{References}

1. Bitwise benchmarks. http://www.cag.lcs.mit.edu/bitwise/bitwise_benchmarks.htm.

2. Gcc compiler. http://gcc.gnu.org/, 2004.

3. Daniel Berlin, David Edelsohn, and Sebastian Pop. High-level loop optimizations for gcc. In The 2004 GCC Developers' Summit, 2004.

4. Preston Briggs, Keith D. Cooper, and Linda Torczon. Improvements to graph coloring register allocation. ACM Transactions on Programming Languages and Systems, 16(3):428-455, May 1994.

5. David Brooks and Margaret Martonosi. Dynamically exploiting narrow width operands to improve processor power and performance. In HPCA '99: Proceedings of the The Fifth International Symposium on High Performance Computer Architecture, page 13, Washington, DC, USA, 1999. IEEE Computer Society.

6. Mihai Budiu, Majd Sakr, Kip Walker, and Seth Copen Goldstein. Bitvalue inference: Detecting and exploiting narrow bitwidth computations. In Euro-Par '00: Proceedings from the 6th International Euro-Par Conference on Parallel Processing, pages 969-979, London, UK, 2000. Springer-Verlag.

7. Gregory J. Chaitin. Register allocation and spilling via graph coloring. In Proceedings of the ACM SIGPLAN '82 Symposium on Compiler Construction, pages 98-105, Jun. 1982.

8. Jack W. Davidson and Sanjay Jinturkar. Memory access coalescing: a technique for eliminating redundant memory accesses. In PLDI '94: Proceedings of the ACM SIGPLAN 1994 conference on Programming language design and implementation, pages 186-195, New York, NY, USA, 1994. ACM Press.

9. Rajiv Gupta, Eduard Mehofer, and Youtao Zhang. A representation for bit section based analysis and optimization. In CC '02: Proceedings of the 11th International Conference on Compiler Construction, pages 62-77, London, UK, 2002. SpringerVerlag. 
10. W. Harrison. Compiler analysis of the value ranges for variables. IEEE Transactions on Software Engineering, (3), May 1977.

11. Johnson Kin, Munish Gupta, and William H. Mangione-Smith. The filter cache: an energy efficient memory structure. In MICRO 30: Proceedings of the 30th annual ACM/IEEE international symposium on Microarchitecture, pages 184-193, Washington, DC, USA, 1997. IEEE Computer Society.

12. Kathleen Knobe and Vivek Sarkar. Array ssa form and its use in parallelization. In POPL '98: Proceedings of the 25th ACM SIGPLAN-SIGACT symposium on Principles of programming languages, pages 107-120, New York, NY, USA, 1998. ACM Press.

13. Kathleen Knobe and Vivek Sarkar. Conditional constant propagation of scalar and array references using array SSA form. In Giorgio Levi, editor, Lecture Notes in Computer Science, 1503, pages 33-56. Springer-Verlag, 1998. Proceedings from the 5th International Static Analysis Symposium.

14. Samuel Larsen and Saman Amarasinghe. Exploiting superword level parallelism with multimedia instruction sets. In PLDI '00: Proceedings of the ACM SIGPLAN 2000 conference on Programming language design and implementation, pages 145156, New York, NY, USA, 2000. ACM Press.

15. Bengu Li and Rajiv Gupta. Bit section instruction set extension of arm for embedded applications. In Proceedings of the international conference on Compilers, architecture, and synthesis for embedded systems, pages 69-78. ACM Press, 2002.

16. Bengu Li, Youtao Zhang, and Rajiv Gupta. Speculative subword register allocation in embedded processors. In Proceedings of the LCPC 2004 Workshop, 2004.

17. Unsal O.S., Wang Z., Koren I., Krishna C.M., and Moritz C.A. On memory behavior of scalars in embedded multimedia systems. In MPI Workshop, ISCA01, 2001.

18. Massimiliano Poletto and Vivek Sarkar. Linear scan register allocation. ACM Transactions on Programming Languages and Systems, 21(5):895-913, 1999.

19. Mark Stephenson, Johnathan Babb, and Saman Amarasinghe. Bitwidth analysis with application to silicon compilation. In ACM SIGPLAN Conference on Programming Language Design and Implementation, Vancouver, British Columbia, June 2000.

20. Sriraman Tallam and Rajiv Gupta. Bitwidth aware global register allocation. In POPL '03: Proceedings of the 30th ACM SIGPLAN-SIGACT symposium on Principles of programming languages, pages 85-96, New York, NY, USA, 2003. ACM Press.

21. Omri Traub, Glenn H. Holloway, and Michael D. Smith. Quality and speed in linear-scan register allocation. In SIGPLAN Conference on Programming Language Design and Implementation, pages 142-151, 1998.

\section{A Appendix}

Theorem 1. The estimates $E_{1}, E_{2}$ and $E_{3}$ are safe i.e., each of $E_{1}, E_{2}$ and $E_{3}$ is greater than or equal to $\operatorname{MIW}(A, B, C)$.

Proof. Let $M I W(A, B, C)=W_{A}+W_{B}+W_{C}$, where $W_{A}, W_{B}$, and $W_{C}$ are contributions of $A, B$, and $C$ to $M I W(A, B, C)$. By definition of $M I W$ it is true that:

$$
W_{A}+W_{B} \leq A_{b}+B_{a}
$$


Similarly, by definition of $N O D E M A X$ it must be the case that:

$$
W_{C} \leq N O D E M A X(C)
$$

Combining the above two equations:

$$
W_{A}+W_{B}+W_{C} \leq A_{b}+B_{a}+\operatorname{NODEMAX}(C)
$$

$\Rightarrow$

$$
E_{1} \geq W_{A}+W_{B}+W_{C}
$$

$E_{1}$ is safe. In a similar fashion we can prove that $E_{2}$ and $E_{3}$ are safe.

Theorem 2. The estimates $E_{4}, E_{5}$ and $E_{6}$ are safe i.e., each of $E_{4}, E_{5}$ and $E_{6}$ is greater than or equal to $\operatorname{MIW}(A, B, C)$.

Proof. By definition of $M I W$,

$$
\begin{aligned}
& A_{b}+B_{a} \geq W_{A}+W_{B} \\
& A_{c}+C_{a} \geq W_{A}+W_{C}
\end{aligned}
$$

$\Rightarrow$

$$
B_{a}+C_{a}+A_{b}+A_{c} \geq W_{B}+W_{C}+2 W_{A}
$$

$\Rightarrow$

$$
B_{a}+C_{a}+\operatorname{MAX}\left(A_{b}, A_{c}\right) \geq W_{B}+W_{C}+W_{A}+W_{A}-\operatorname{MIN}\left(A_{b}, A_{c}\right)
$$

$\Rightarrow$ If $W_{A} \geq \operatorname{MIN}\left(A_{b}, A_{c}\right)$, then

$$
B_{a}+C_{a}+M A X\left(A_{b}, A_{c}\right) \geq W_{A}+W_{B}+W_{C}
$$

$\Rightarrow$ If $W_{A} \geq \operatorname{MIN}\left(A_{b}, A_{c}\right)$, then

$$
E_{4} \geq W_{A}+W_{B}+W_{C}
$$

Whether $W_{A} \geq \operatorname{MIN}\left(A_{b}, A_{c}\right)$ is obtained the following way.

If $E_{\text {min }}=E_{a}\left(E_{\text {min }}\right.$ and $E_{a}$ are estimates in Figure 7 of [20] $)$, and $E_{4} \geq E_{\text {min }}$ then estimate $E_{4}$ can be used, because the following two things can happen:

- if $E_{\text {min }} \geq M I W(A, B, C)$ then $E_{4} \geq E_{\text {min }} \geq M I W(A, B, C) . E_{4}$ is safe.

- if $E_{\text {min }}<M I W(A, B, C)$ then $B_{c}+C_{b}+\max \left(A_{b}, A_{c}\right)<W_{A}+W_{B}+W_{C}$. By definition of $M I W, B_{c}+C_{b}<W_{B}+W_{C}$. This implies $W_{A}>M A X\left(A_{b}, A_{c}\right)$ $\geq \operatorname{MIN}\left(A_{b}, A_{c}\right) . E_{4}$ is safe.

Similarly, $E_{5}$ and $E_{6}$ can be proven to be safe. 